PROCEEDINGS OF THE

AMERICAN MATHEMATICAL SOCIETY

Volume 127, Number 12, Pages 3615-3622

S 0002-9939(99)04955-2

Article electronically published on May 13, 1999

\title{
DECOMPOSING SYMMETRICALLY CONTINUOUS AND SIERPIŃSKI-ZYGMUND FUNCTIONS INTO CONTINUOUS FUNCTIONS
}

\author{
KRZYSZTOF CIESIELSKI
}

(Communicated by Alan Dow)

\begin{abstract}
In this paper we will investigate the smallest cardinal number $\kappa$ such that for any symmetrically continuous function $f: \mathbb{R} \rightarrow \mathbb{R}$ there is a partition $\left\{X_{\xi}: \xi<\kappa\right\}$ of $\mathbb{R}$ such that every restriction $f \mid X_{\xi}: X_{\xi} \rightarrow \mathbb{R}$ is continuous. The similar numbers for the classes of Sierpiński-Zygmund functions and all functions from $\mathbb{R}$ to $\mathbb{R}$ are also investigated and it is proved that all these numbers are equal. We also show that $\operatorname{cf}(\mathfrak{c}) \leq \kappa \leq \mathfrak{c}$ and that it is consistent with ZFC that each of these inequalities is strict.
\end{abstract}

\section{Preliminaries}

Our notation and terminology is standard and follows [5]. In particular, $|X|$ will stand for the cardinality of $X$. For a cardinal number $\kappa$ we will write $\operatorname{cf}(\kappa)$ for its cofinality. We also define $[X]^{\kappa}=\{Y \subseteq X:|Y|=\kappa\}$. The definition of $[X]^{<\kappa}$ is similar. The cardinality of the set $\mathbb{R}$ of real numbers is denoted by $\mathfrak{c}$. The functions are identified with their graphs. The class of all function from a set $X$ into a set $Y$ is denoted by $Y^{X}$.

For $Z \subset \mathbb{R}$ and a cardinal number $\kappa \leq \mathfrak{c}$ let $\Pi_{\kappa}(Z)$ denote the family of all coverings of $Z$ with at most $\kappa$ many sets. We will write $\Pi_{\kappa}$ for $\Pi_{\kappa}(\mathbb{R})$. In [4] the authors considered the following cardinal decomposition function for arbitrary families $\mathcal{F} \subset \mathbb{R}^{Z}$, with $Z \subset \mathbb{R}$, and $\mathcal{G} \subset \bigcup\left\{\mathbb{R}^{X}: X \subset Z\right\}$ :

$$
\operatorname{dec}(\mathcal{F}, \mathcal{G})=\min \left(\left\{\kappa \leq \mathfrak{c}:(\forall f \in \mathcal{F})\left(\exists \mathcal{X} \in \Pi_{\kappa}(Z)\right)(\forall X \in \mathcal{X})(f\lceil X \in \mathcal{G})\} \cup\left\{\mathfrak{c}^{+}\right\}\right) .\right.
$$

In particular, if $\mathcal{C}$ stands for the family of all continuous functions from a subset of $\mathbb{R}$ into $\mathbb{R}$, then

$$
f: \mathbb{R} \rightarrow \mathbb{R} \text { is countable continuous if and only if } \operatorname{dec}(\{f\}, \mathcal{C}) \leq \omega .
$$

In [4] the authors considered the values of $\operatorname{dec}\left(\mathcal{B}_{\beta}, \mathcal{B}_{\alpha}\right)$ for $\alpha<\beta<\omega_{1}$, where $\mathcal{B}_{\alpha}$ stands for the functions of $\alpha$-th Baire class. In particular, they proved that

$$
\operatorname{cov}(\mathcal{M}) \leq \operatorname{dec}\left(\mathcal{B}_{1}, \mathcal{C}\right) \leq d
$$

Received by the editors November 23, 1997 and, in revised form, February 18, 1998.

1991 Mathematics Subject Classification. Primary 26A15; Secondary $03 E 35$.

Key words and phrases. Decomposition number, symmetrically continuous functions, Sierpiński-Zygmund functions.

The author was partially supported by NATO Collaborative Research Grant CRG 950347 and 1996/97 West Virginia University Senate Research Grant.

(C)1999 American Mathematical Society 
where $\operatorname{cov}(\mathcal{M})$ is the smallest cardinality of a covering of $\mathbb{R}$ by meager sets, and $d$, the dominating number, is the smallest cardinality of a dominating family $D \subset \omega^{\omega}$, that is, such that for every $f \in \omega^{\omega}$ there exists $g \in D$ such that $f \leq^{*} g$. Moreover, in papers [12] and [11] it has been proved that each of these inequalities can be strict.

There are also some interesting results concerning the value of $\operatorname{dec}(\mathcal{C}, \mathcal{D})$, where $\mathcal{D}$ is the class of all (partial) differentiable functions. It has been proved by Morayne [13, Theorem 6.1] that $\operatorname{dec}(\mathcal{C}, \mathcal{D}) \geq \operatorname{cov}(\mathcal{M})$, while Steprāns [13] proved that it is consistent with ZFC that $\operatorname{dec}(\mathcal{C}, \mathcal{D})<\mathfrak{c}$.

For more information on the subject see also a survey paper [6].

In this paper we will examine the numbers $\operatorname{dec}(\mathcal{F}, \mathcal{C})$, where $\mathcal{F}$ is one of the following three classes:

$\mathbb{R}^{\mathbb{R}}$ of all functions from $\mathbb{R}$ to $\mathbb{R}$;

$\mathrm{SZ}(X)$ of all Sierpinski-Zygmund functions from $X \subseteq \mathbb{R}$ into $\mathbb{R}$, that is, all $f: X \rightarrow \mathbb{R}$ whose restrictions $f \nmid Y$ are discontinuous for all subsets $Y$ of $X$ of cardinality continuum $\mathfrak{c}$ (we will write $\mathrm{SZ}$ for $\mathrm{SZ}(\mathbb{R})$ );

Sc of all symmetrically continuous functions $f: \mathbb{R} \rightarrow \mathbb{R}$, that is, such that for every $x \in \mathbb{R}$ we have

$$
\lim _{h \rightarrow 0^{+}} f(x-h)-f(x+h)=0 .
$$

Now, since $\mathcal{F} \subset \mathcal{F}^{\prime}$ implies $\operatorname{dec}(\mathcal{F}, \mathcal{G}) \leq \operatorname{dec}\left(\mathcal{F}^{\prime}, \mathcal{G}\right)$ and a Sierpiński-Zygmund function $f: X \rightarrow \mathbb{R}$, with $X \in[\mathbb{R}]^{\mathfrak{c}}$, cannot be covered by less than $\operatorname{cf}(\mathfrak{c})$-many continuous functions, we obtain immediately the following inequalities:

$$
\operatorname{cf}(\mathfrak{c}) \leq \operatorname{dec}(\{f\}, \mathcal{C}) \leq \operatorname{dec}(\mathrm{SZ}, \mathcal{C}) \leq \operatorname{dec}\left(\mathbb{R}^{\mathbb{R}}, \mathcal{C}\right) \leq \mathfrak{c}
$$

for any $f \in \mathrm{SZ}(X)$ with $X \in[\mathbb{R}]^{\mathfrak{c}}$. Also, in [7] the authors proved $\operatorname{dec}(\mathrm{Sc}, \mathcal{C})>\omega$ by showing that there exists an $f \in \operatorname{Sc}$ such that $f\left\lceil X \in \mathrm{SZ}(X)\right.$ for some $X \in[\mathbb{R}]^{\mathrm{c}}$. This clearly implies that

$$
\operatorname{cf}(\mathfrak{c}) \leq \operatorname{dec}(\{f \mid X\}, \mathcal{C}) \leq \operatorname{dec}(\operatorname{Sc}, \mathcal{C}) \leq \operatorname{dec}\left(\mathbb{R}^{\mathbb{R}}, \mathcal{C}\right) \leq \mathfrak{c} .
$$

The main results of this paper are the following three theorems refining the results of (1) and (2).

Theorem 1.1 (Ciesielski, Szyszkowski $\left.{ }^{1}\right) \cdot \operatorname{dec}(\operatorname{Sc}, \mathcal{C})=\operatorname{dec}\left(\mathbb{R}^{\mathbb{R}}, \mathcal{C}\right)$.

Theorem 1.2. $\operatorname{dec}(\mathrm{SZ}, \mathcal{C})=\operatorname{dec}\left(\mathbb{R}^{\mathbb{R}}, \mathcal{C}\right)$.

Theorems 1.1 and 1.2 immediately imply the following corollary.

Corollary 1.1. $\operatorname{cf}(\mathfrak{c}) \leq \operatorname{dec}(\operatorname{SZ}, \mathcal{C})=\operatorname{dec}(\operatorname{Sc}, \mathcal{C})=\operatorname{dec}\left(\mathbb{R}^{\mathbb{R}}, \mathcal{C}\right) \leq \mathfrak{c}$.

The next theorem tells us that neither of the inequalities in Corollary 1.1 can be replaced by the equation.

Theorem 1.3. Let $\lambda$ be a cardinal number with uncountable cofinality. Then

(a) it is relatively consistent with $Z F C$ that $\mathfrak{c}=\lambda=\operatorname{dec}\left(\mathbb{R}^{\mathbb{R}}, \mathcal{C}\right)$; and

(b) it is relatively consistent with $Z F C$ that $\mathfrak{c}=\lambda$ and $\operatorname{dec}\left(\mathbb{R}^{\mathbb{R}}, \mathcal{C}\right)=\operatorname{cf}(\lambda)$.

\footnotetext{
${ }^{1}$ This result was obtained during Mr. Szyszkowski's work on his doctoral degree under the author's supervision. It will most likely become a part of Mr. Szyszkowski's dissertation.
} 
The statement of the theorem is not very precise. (For example, we can't have $\kappa=\mathfrak{c}^{+}$in the conclusion of the theorem.) Our interpretation of it is that if $\lambda$ satisfies the assumption of the theorem in a model $M$ of ZFC satisfying the Generalized Continuum Hypothesis GCH, then we can find an extension of $M$ to another ZFC model in which all the cardinals are the same, have the same cofinalities, and either (a) or (b) holds. In particular Theorem 1.3 implies the following corollary.

Corollary 1.2. (a) It is consistent with $Z F C$ that $\operatorname{cf}(\mathfrak{c})<\mathfrak{c}=\operatorname{dec}\left(\mathbb{R}^{\mathbb{R}}, \mathcal{C}\right)$.

(b) It is consistent with $Z F C$ that $\operatorname{dec}\left(\mathbb{R}^{\mathbb{R}}, \mathcal{C}\right)=\operatorname{cf}(\mathfrak{c})<\mathfrak{c}$.

Theorems 1.1, 1.2, and 1.3 will be proved in the next sections. We will finish this section with the following open problem.

Problem 1.1. Is it consistent with ZFC that $\operatorname{cf}(\mathfrak{c})<\operatorname{dec}\left(\mathbb{R}^{\mathbb{R}}, \mathcal{C}\right)<\mathfrak{c}$ ?

\section{Proof of Theorem 1.1}

In order to prove the theorem we will show the following fact that is of independent interest.

Proposition 2.1. There exists a perfect set $P \subset \mathbb{R}$ with the property that for every $f_{0}: P \rightarrow[0,1]$ there is a symmetrically continuous function $f: \mathbb{R} \rightarrow \mathbb{R}$ extending $f_{0}$.

The proof is a compilation of the results contained in [7] and [3]. In particular, if $C(f)$ stands for the set of points of continuity of a function $f: \mathbb{R} \rightarrow \mathbb{R}$ and $D(f)=\mathbb{R} \backslash C(f)$, then the following lemma is a rephrasing of [7, Lemma 2.1] (used with $\left.\left\{A_{\alpha}\right\}_{\alpha \in \mathcal{A}}=\{2 D(h)+y: y \in X\}\right)$.

Lemma 2.1. Let $h: \mathbb{R} \rightarrow \mathbb{R}$ and $X \subset D(h)$ be such that $h$ is symmetrically continuous and

(i) $C(h)=h^{-1}(0)$,

(ii) $D(h)$ is an additive subgroup of $\mathbb{R}$,

(iii) $(2 D(h)+x) \cap(2 D(h)+y)=\emptyset$ for every distinct $x, y \in X$.

Then for every map $r: X \rightarrow[0,1]$ the function

$$
f(x)=h(x) \cdot \sum_{y \in X} r(y) \chi_{2 D(h)+y}(x)
$$

is symmetrically continuous.

Now, in [3] (in the proof of Theorem 1) Chlebík shows that the function $h: \mathbb{R} \rightarrow$ $[0,1]$ defined by formula

$$
h(x)=\lim _{m \rightarrow \infty}\left(1+\sum_{n=1}^{m}\left|\frac{1}{n} \sin 3^{n} x\right|\right)^{-1}
$$

is upper semicontinuous, symmetrically continuous, and satisfies (i) and (ii) from Lemma 2.1. Towards the construction of a set $X$ he defines the following.

He takes an arbitrary linear basis $\mathcal{H} \subset(0,1]$ of $\mathbb{R}$ over $\mathbb{Q}$ with $1 \in \mathcal{H}$, puts $\Lambda=\mathcal{H} \backslash\{1\}$, and defines $X=\pi \cdot \psi[\Lambda]$ for a continuous injection $\psi:(0,1] \backslash \mathbb{Q} \rightarrow(0,1)$ given by the formula

$$
\psi(x)=\sum_{k=1}^{\infty} \mu_{k} \cdot 3^{-\left(2^{k}+1\right)}
$$


where $x=\sum_{k=1}^{\infty} \mu_{k} \cdot 2^{-k}$, with $\mu_{k} \in\{0,1\}$, is the unique binary representation of $x$. Chlebík proves also that $X \subset D(h)$ and that

$$
2 D(h)+H_{1} \neq 2 D(h)+H_{2} \quad \text { for every distinct } H_{1}, H_{2} \subset X,
$$

while this last property is used in [7, Lemma 2.4] to prove that $X$ satisfies (iii) of Lemma 2.1.

Thus, $h$ and $X$ satisfy the assumptions of Lemma 2.1. However, to prove Proposition 2.1 we need also two additional facts that

(iv) $h$ is of Baire class one (as upper semicontinuous), and that

(v) $X$ is a continuous image of $\mathcal{H} \backslash\{1\}$, where $\mathcal{H}$ is an arbitrary linear basis of $\mathbb{R}$ over $\mathbb{Q}$ with $1 \in \mathcal{H} \subset(0,1]$.

Now, take a perfect set $K \subset[0,1]$ which is linearly independent over $\mathbb{Q}$. (See e.g. [8, Theorem 2, Ch. XI sec. 7].) Decreasing it, if necessary, we can assume that 1 is linearly independent of $K$. (If it is not, take a finite subset $A$ of $K$ which spans 1 and replace $K$ by its perfect subset disjoint with $A$.) Thus, there exists a linear basis $\mathcal{H} \subset(0,1]$ of $\mathbb{R}$ over $\mathbb{Q}$ such that $\{1\} \cup K \subset \mathcal{H}$. In particular, $X_{0}=\psi[K]$ is a perfect subset of $X=\psi[\mathcal{H} \backslash\{1\}]$, so it satisfies (iii).

Now, $h\left\lceil X_{0}\right.$ has a point of continuity, say $x_{0} \in X_{0}$, since $h$ is of Baire class one. (See e.g. [2].) Since $x_{0} \in X \subset D(h)=\mathbb{R} \backslash h^{-1}(0)$, we have $h\left(x_{0}\right) \neq 0$. Thus, we can take a perfect subset $P$ of $X_{0}$ for which $h[P] \subset[b, 1]$ for some $b>0$. We will show that $P$ satisfies Proposition 2.1.

To see it note that by Lemma 2.1 used with $X=P$ the function

$$
f(x)=\frac{1}{b} h(x) \cdot \sum_{y \in P} r(y) \chi_{2 D(h)+y}(x)
$$

is symmetrically continuous for any function $r: P \rightarrow[0,1]$. Moreover, $f(x)=$ $\frac{1}{b} h(x) \cdot r(x)$ for every $x \in P$ since $x \in 2 D(h)+x$. (D(h) is a group.) Thus defining $r$ by

$$
r(x)=\frac{b}{h(x)} \cdot f_{0}(x) \leq f_{0}(x)
$$

we obtain that $r: P \rightarrow[0,1]$ and

$$
f(x)=\frac{1}{b} h(x) \cdot r(x)=f_{0}(x)
$$

for every $x \in P$. This finishes the proof of Proposition 2.1.

Proof of Theorem 1.1. Clearly $\operatorname{dec}(\operatorname{Sc}, \mathcal{C}) \leq \operatorname{dec}\left(\mathbb{R}^{\mathbb{R}}, \mathcal{C}\right)$. To prove the other inequality take an arbitrary $g \in \mathbb{R}^{\mathbb{R}}$ and let $\kappa=\operatorname{dec}(\{g\}, \mathcal{C})$. It is enough to prove that

$$
\kappa \leq \operatorname{dec}(\mathrm{Sc}, \mathcal{C}) \text {. }
$$

If $\kappa<\omega_{1}$, then (3) follows from $\operatorname{cf}(\mathfrak{c}) \leq \operatorname{dec}(\mathrm{Sc}, \mathcal{C})$. So, we can assume that $\kappa$ is uncountable. Also, if $h$ is a homeomorphism between $\mathbb{R}$ and $(0,1)$, then it is easy to see that $\operatorname{dec}(\{g\}, \mathcal{C})=\operatorname{dec}(\{h \circ g\}, \mathcal{C})$. So, we can assume that $g: \mathbb{R} \rightarrow(0,1)$. Moreover, if $\mathcal{N}=\mathbb{R} \backslash \mathbb{Q}$, then $\kappa=\operatorname{dec}(\{g\}, \mathcal{C})=\operatorname{dec}(\{g\lceil\mathcal{N}\}, \mathcal{C})$, since $\kappa$ is uncountable.

Let $h$ be a homeomorphism between $\mathcal{N}$ and a subset $M$ of $P$, where $P$ is from Proposition 2.1, and define $f_{0}: M \rightarrow[0,1]$ by $f_{0}=g \circ h^{-1}$. Once again it is easy 
to see that $\kappa=\operatorname{dec}(\{g\}, \mathcal{C})=\operatorname{dec}\left(\left\{f_{0}\right\}, \mathcal{C}\right)$. Now, if $f$ is a symmetrically continuous function extending $f_{0}$, which exists by Proposition 2.1, then

$$
\kappa=\operatorname{dec}\left(\left\{f_{0}\right\}, \mathcal{C}\right) \leq \operatorname{dec}(\{f\}, \mathcal{C}) \leq \operatorname{dec}(\operatorname{Sc}, \mathcal{C})
$$

proving (3). The proof of Theorem 1.1 is complete.

\section{Proof of Theorem 1.2}

The inequality $\operatorname{dec}(\mathrm{SZ}, \mathcal{C}) \leq \operatorname{dec}\left(\mathbb{R}^{\mathbb{R}}, \mathcal{C}\right)$ follows from (1). To prove the other inequality let $\kappa=\operatorname{dec}\left(\mathbb{R}^{\mathbb{R}}, \mathcal{C}\right)$. We will prove that

$$
\kappa \leq \operatorname{dec}(\mathrm{SZ}, \mathcal{C}) .
$$

First note that there exists an $f \in \mathbb{R}^{\mathbb{R}}$ such that

$$
\operatorname{dec}(\{f\}, \mathcal{C})=\operatorname{dec}\left(\mathbb{R}^{\mathbb{R}}, \mathcal{C}\right)=\kappa .
$$

Indeed, if $\kappa$ is a successor cardinal, then (5) is obvious. So assume that $\kappa$ is a limit cardinal. Clearly for every $\xi<\kappa$ there exists an $f_{\xi}: \mathbb{R} \rightarrow \mathbb{R}$ such that $\operatorname{dec}\left(\left\{f_{\xi}\right\}, \mathcal{C}\right) \geq|\xi|$. Then $\operatorname{dec}\left(\left\{f_{\xi}\right\}, \mathcal{C}\right)=\operatorname{dec}\left(\left\{f_{\xi}\lceil\mathcal{N}\}, \mathcal{C}\right)\right.$ for every $\omega_{1} \leq \xi<\lambda$, where $\mathcal{N}=\mathbb{R} \backslash \mathbb{Q}$. Take a family $\left\{\mathcal{N}_{\xi}: \xi<\kappa\right\}$ of pairwise disjoint subsets of $\mathbb{R}$ homeomorphic to $\mathcal{N}$ and let $h_{\xi}: \mathcal{N}_{\xi} \rightarrow \mathcal{N}$ be the homeomorphisms. It is easy to see that $\operatorname{dec}\left(\left\{f_{\xi} \mid \mathcal{N}\right\}, \mathcal{C}\right)=\operatorname{dec}\left(f_{\xi} \circ h_{\xi}, \mathcal{C}\right)$. Thus, if $f \in \mathbb{R}^{\mathbb{R}}$ is any extension of $\bigcup_{\xi<\kappa} f_{\xi} \circ h_{\xi}$, then

$$
\kappa=\sup _{\omega_{1} \leq \xi<\kappa}|\xi| \leq \sup _{\omega_{1} \leq \xi<\kappa} \operatorname{dec}\left(\left\{f_{\xi}\right\}, \mathcal{C}\right) \leq \operatorname{dec}(\{f\}, \mathcal{C}) \leq \operatorname{dec}\left(\mathbb{R}^{\mathbb{R}}, \mathcal{C}\right)=\kappa
$$

proving (5).

Now, if $\kappa \leq \operatorname{cf}(\mathfrak{c})$, then (4) follows from (1). So, we will be assuming that

$$
\kappa>\operatorname{cf}(\mathfrak{c}) .
$$

Then, by $(1), \operatorname{cf}(\mathfrak{c})<\mathfrak{c}$.

Let $f \in \mathbb{R}^{\mathbb{R}}$ be such that $\operatorname{dec}(\{f\}, \mathcal{C})=\kappa$ and let $\left\{X_{\xi}: \xi<\operatorname{cf}(\mathfrak{c})\right\}$ be a partition of $\mathbb{R}$ such that $\left|X_{\xi}\right|<\mathfrak{c}$ for every $\xi<\operatorname{cf}(\mathfrak{c})$. Notice that

$$
\operatorname{dec}(\{f\}, \mathcal{C}) \leq \sup _{\xi<\operatorname{cf}(\mathfrak{c})} \operatorname{dec}\left(\left\{f \uparrow X_{\xi}\right\}, \mathcal{C}\right) .
$$

To see it let $\lambda=\sup _{\xi<\operatorname{cf}(\mathfrak{c})} \operatorname{dec}\left(\left\{f \mid X_{\xi}\right\}, \mathcal{C}\right)$ and for every $\xi<\operatorname{cf}(\mathfrak{c})$ choose $\mathcal{X}_{\xi} \in$ $\Pi_{\lambda}\left(X_{\xi}\right)$ such that $f \nmid X \in \mathcal{C}$ for every $X \in \mathcal{X}_{\xi}$. Then the family $\mathcal{X}=\bigcup_{\xi<\mathrm{cf}(\mathfrak{c})} \mathcal{X}_{\xi}$ has cardinality at most $\operatorname{cf}(\mathfrak{c}) \otimes \lambda$. Therefore, $\kappa=\operatorname{dec}(\{f\}, \mathcal{C}) \leq \operatorname{cf}(\mathfrak{c}) \otimes \lambda$. Hence, by $(6), \lambda \geq \kappa=\operatorname{dec}(\{f\}, \mathcal{C})$ proving $(7)$.

To finish the proof, let $\left\{g_{\xi}: \xi<\mathfrak{c}\right\}$ be an enumeration of all continuous functions from a $G_{\delta}$ subset of $\mathbb{R}$ into $\mathbb{R}$ and let $\left\langle\lambda_{\xi}: \xi<\operatorname{cf}(\mathfrak{c})\right\rangle$ be an increasing sequence cofinal with $\mathfrak{c}$. For every $\xi<\operatorname{cf}(\mathfrak{c})$ choose a number $b_{\xi} \in \mathbb{R}$ such that

$$
\left(b_{\xi}+f\left[X_{\xi}\right]\right) \cap \bigcup_{\zeta<\lambda_{\xi}} g_{\zeta}\left[X_{\xi}\right]=\emptyset .
$$

Such a number can be found since the sets $f\left[X_{\xi}\right]$ and $\bigcup_{\zeta<\lambda_{\xi}} g_{\zeta}\left[X_{\xi}\right]$ have cardinality less than $\mathfrak{c}$. Let

$$
g=\bigcup_{\xi<\mathfrak{c}}\left(b_{\xi}+f \uparrow X_{\xi}\right)
$$


and note that, by (8), $g(x)=b_{\xi}+f(x) \neq g_{\zeta}(x)$ for any $x \in X_{\xi}$ and $\zeta<\lambda_{\xi}$. In particular $g \in \mathrm{SZ}$. Therefore, by (7),

$$
\operatorname{dec}(\{f\}, \mathcal{C}) \leq \sup _{\xi<\operatorname{cf}(\mathfrak{c})} \operatorname{dec}\left(\left\{f \uparrow X_{\xi}\right\}, \mathcal{C}\right)=\sup _{\xi<\operatorname{cf}(\mathfrak{c})} \operatorname{dec}\left(\left\{b_{\xi}+f \uparrow X_{\xi}\right\}, \mathcal{C}\right) \leq \operatorname{dec}(\{g\}, \mathcal{C})
$$

and so

$$
\kappa \leq \operatorname{dec}(\{g\}, \mathcal{C}) \leq \operatorname{dec}(\mathrm{SZ}, \mathcal{C}) .
$$

This finishes the proof of Theorem 1.2.

\section{Proof of Theorem 1.3}

Part (a) of Theorem 1.3 holds in a Cohen model obtained by adding $\lambda=\mathfrak{c}$ Cohen reals. This follows from the fact, proved by G. Gruenhage (see Recław $[9$, Theorem 4]) and S. Shelah [10] (see also [6]), that in such a model there exists an $f: \mathbb{R} \rightarrow \mathbb{R}$ for which $f\lceil X$ is discontinuous for every uncountable $X \subset \mathbb{R}$.

The fact that Theorem 1.3(a) holds in a Cohen model can also be easily proved directly. (Some difficulty in the result of Gruenhage and Shelah comes from the fact that their function is defined on the entire real line. For our proof, however, it is enough to have a partial function $f$ defined on a set of cardinality $\mathfrak{c}$ with the same property.) Simply, let $\left\{x_{\langle\xi, i\rangle}:\langle\xi, i\rangle \in \lambda \times 2\right\}$ be a one-to-one enumeration of the Cohen reals and define $f$ on $X=\left\{x_{\langle\xi, 0\rangle}: \xi<\lambda\right\}$ by $f\left(x_{\langle\xi, 0\rangle}\right)=x_{\langle\xi, 1\rangle}$. Then $f$ has the desired property. ${ }^{2}$

In the proof of part (b) of Theorem 1.3 we will need the following lemma, which is an easy variation of a result in Baldwin [1] that under the Martin's Axiom MA for every function $f: \mathbb{R} \rightarrow \mathbb{R}$ and every infinite $\kappa<\mathfrak{c}$ there exists a $\kappa$-dense set $X \subset \mathbb{R}$ such that $f \uparrow X$ is continuous.

Lemma 4.1. If $M A$ holds, then for every $X \subset \mathbb{R}$ with cardinality less than $\mathfrak{c}$ and for every $f: X \rightarrow \mathbb{R}$ there exists a countable partition $\left\{X_{n}: n<\omega\right\}$ of $X$ such that $f \uparrow X_{n}$ is continuous for every $n<\omega$.

Proof. Let $D$ be a countable dense subset of $[-\infty, \infty] \backslash X$ which contains $\{-\infty, \infty\}$ and let $\mathcal{S}$ be the family of all finite unions $\bigcup_{i=0}^{n}\left(a_{i}, b_{i}\right) \times\left(c_{i}, d_{i}\right)$, where $a_{i}, b_{i}, c_{i}, d_{i} \in$ $D, a_{i}<b_{i}, c_{i}<d_{i}$, and the intervals $\left\{\left(a_{i}, b_{i}\right)\right\}_{i=0}^{n}$ form a disjoint cover of $\mathbb{R} \backslash D$. Moreover, for $k>0$ let $\mathcal{S}_{k}$ be the family of these unions $\bigcup_{i=0}^{n}\left(a_{i}, b_{i}\right) \times\left(c_{i}, d_{i}\right)$ from $\mathcal{S}$ for which $\left(a_{i}, b_{i}\right)$ and $\left(c_{i}, d_{i}\right)$ have lengths less than $1 / k$ for every $i$ with $\left(a_{i}, b_{i}\right) \cap(-k, k) \neq \emptyset$.

Consider the forcing

$$
R_{f}=\left\{\langle A, S\rangle: A \in[X]^{<\omega} \& f \uparrow A \subset S \in \mathcal{S}\right\}
$$

ordered by $\langle A, S\rangle \leq\langle B, T\rangle$ if $B \subset A$ and $S \subset T$. Define $P_{f}$ as a finite support product of forcings $R_{f}$, that is, $P_{f}$ is the set of all sequences $\left\langle\left\langle A_{j}, S_{j}\right\rangle: j<\omega\right\rangle$ from $\left(R_{f}\right)^{\omega}$ for which $\left\langle A_{j}, S_{j}\right\rangle=\left\langle\emptyset, \mathbb{R}^{2}\right\rangle$ for all but finitely many $j$ 's.

It is easy to see that $P_{f}$ is ccc (in fact, it is $\sigma$-centered) since the family $\mathcal{S}$ is countable and any conditions from $R_{f}$ with the same second coordinate are compatible. Next notice that the following subsets of $P_{f}$ are dense for every $x \in X$

\footnotetext{
${ }^{2}$ It has been pointed by the referee that the existence of such a partial function $f$ follows from the existence Lusin set (also Sierpinski set) of size continuum. Simply, choose $f$ of size continuum in the Lusin subset of the plane of size continuum.
} 
and $i<k<\omega$ :

$$
\begin{gathered}
D_{x}=\left\{\left\langle A_{j}, S_{j}\right\rangle_{j<\omega} \in P_{f}: x \in \bigcup_{j<\omega} A_{j}\right\}, \\
E_{i, k}=\left\{\left\langle A_{j}, S_{j}\right\rangle_{j<\omega} \in P_{f}: S_{i} \in \mathcal{S}_{k}\right\} .
\end{gathered}
$$

Let $\mathcal{G}=\left\{D_{x}: x \in X\right\} \cup\left\{E_{i, k}: i<k<\omega\right\}$ and let $\mathcal{F}$ be a $\mathcal{G}$-generic filter in $P_{f}$. For $i<\omega$ we put

$$
X_{i}=\bigcup\left\{A_{i}:\left\langle A_{j}, S_{j}\right\rangle_{j<\omega} \in \mathcal{F}\right\} .
$$

Then the sets $D_{x}$ guarantee that $\bigcup_{i<\omega} X_{i}=X$, while the sets $E_{i, k}$ force that each restriction $f \uparrow X_{i}$ is continuous.

Now, to prove Theorem 1.3(b) start with a model $M$ of $\mathrm{ZFC}+\mathrm{GCH}$ and take a cardinal $\lambda$ with uncountable cofinality.

If $\operatorname{cf}(\lambda)=\lambda$, then (b) holds in a model from part (a). Thus we will assume that $\operatorname{cf}(\lambda)<\lambda$. Let $\left\{\lambda_{\xi}: \xi<\operatorname{cf}(\lambda)\right\}$ be an increasing sequence cofinal with $\lambda$ such that each $\lambda_{\xi}$ is a cardinal successor. Define $P$ as a finite support iteration of forcings $M_{\xi}$, where each $M_{\xi}$ is a standard ccc forcing adding the Martin's Axiom over the previous model and making $\mathfrak{c}=\lambda_{\xi}$. Let $G$ be an $M$-generic filter over $P$. We claim that (b) holds in $M[G]$.

Checking that $\mathfrak{c}=\lambda$ in $M[G]$ is routine. To see that $\operatorname{dec}\left(\mathbb{R}^{\mathbb{R}}, \mathcal{C}\right)=\operatorname{cf}(\lambda)$ it is enough to show that $\operatorname{dec}(\{f\}, \mathcal{C}) \leq \operatorname{cf}(\lambda)$ for every $f \in \mathbb{R} \rightarrow \mathbb{R}$. So fix $f \in \mathbb{R}^{\mathbb{R}}$ from $M[G]$ and let $\hat{f}$ be a $P$-name for $f$. For $\xi<\operatorname{cf}(\lambda)$ let $X_{\xi}$ be the set of all $x \in \mathbb{R}$ for which the value of $\hat{f}(x)$ is already decided in the model $M\left[G \cap P_{\xi}\right]$, where $P_{\xi}$ is the iteration of forcings $M_{\zeta}$ up to $\xi$. Then $\mathbb{R}=\bigcup_{\xi<\operatorname{cf}(\lambda)} X_{\xi}$ and, by Lemma 4.1 , in $M\left[G \cap P_{\xi+1}\right]$ there is a cover $\left\{X_{\xi}^{n}: n<\omega\right\}$ of $X_{\xi}$ such that each function $f \uparrow X_{\xi}^{n}$ is continuous. Then functions $\left\{f \uparrow X_{\xi}^{n}: \xi<\operatorname{cf}(\lambda) \& n<\omega\right\}$ witness $\operatorname{dec}(\{f\}, \mathcal{C}) \leq \operatorname{cf}(\lambda)$ in $M[G]$, finishing the proof.

\section{REFERENCES}

[1] S. Baldwin, Martin's axiom implies a stronger version of Blumberg's theorem, Real Anal. Exchange 16 (1990-91), 67-73. MR 92b:26005

[2] A. M. Bruckner, Differentiation of Real Functions, CMR Series vol. 5, Amer. Math. Soc., 1994. MR 94m:26001

[3] M. Chlebík, There are $2^{\mathfrak{c}}$ symmetrically continuous functions, Proc. Amer. Math. Soc. 113 (1991), 683-688. MR 92b:26006

[4] J. Cichoń, M. Morayne, J. Pawlikowski, S. Solecki, Decomposing Baire functions, J. Symbolic Logic 56 (1991), 1273-1283. MR 92j:04001

[5] K. Ciesielski, Set Theory for the Working Mathematician, London Math. Soc. Student Texts 39, Cambridge Univ. Press 1997. CMP 98:02

[6] K. Ciesielski, Set Theoretic Real Analysis, J. Appl. Anal. 3(2) (1997), 143-190. (Preprint* available. ${ }^{3}$ )

[7] K. Ciesielski, M. Szyszkowski, A symmetrically continuous function which is not countably continuous, Real Anal. Exchange 22 (1996-97), 428-432. (Preprint* available.) MR 97h:26002

[8] M. Kuczma, An Introduction to the Theory of Functional Equations and Inequalities, Polish Scientific Publishers PWN, Warsaw 1985. MR 86i:39008

[9] I. Recław, Restrictions to continuous functions and Boolean algebras, Proc. Amer. Math. Soc. 118 (1993), 791-796.) MR 93i:26003

\footnotetext{
${ }^{3}$ Preprints marked by * are available in electronic form. They can be accessed from the Set Theoretic Analysis Web Page: http://www.math.wvu.edu/homepages/kcies/STA/STA.html.
} 
[10] S. Shelah, Possibly every real function is continuous on a non-meagre set, Publications de L'Institute Mathematique - Beograd, Nouvelle Serie 57(71) (1995), 47-60.

[11] S. Shelah, J. Steprāns, Decomposing Baire class 1 functions into continuous functions, Fund. Math. 145 (1994), 171-180. MR 97c:03122

[12] J. Steprāns, A very discontinuous Borel function, J. Symbolic Logic 58 (1993), 1268-1283. MR 95c:03120

[13] J. Steprāns, Decomposing with smooth sets, Trans. Amer. Math. Soc., to appear. (Preprint* available.) CMP 98:02

Department of Mathematics, West Virginia University, Morgantown, West Virginia 26506-6310

E-mail address: KCies@wvnvms.wvnet.edu 